\title{
Perturbation analysis of weakly discrete kinks
}

\author{
S. Flach* and K. Kladko \\ Max-Planck-Institut für Physik komplexer Systeme \\ Noethnitzer Str. 38, D-01187 Dresden, Germany
}

\begin{abstract}
We present a perturbation theory of kink solutions of discrete Klein-Gordon chains. The unperturbed solutions correspond to the kinks of the adjoint partial differential equation. The perturbation theory is based on a reformulation of the discrete chain problem into a partial differential equation with spatially modulated mass density. The first order corrections to the kink solutions are obtained analytically and are shown to agree with exact numerical results. We discuss the problem of calculating the Peierls-Nabarro barrier.

PACS number(s): 03.20.+i ; 03.40.-t ; 63.20.Ry
\end{abstract}

* email: flach@idefix.mpipks-dresden.mpg.de 


\section{INTRODUCTION}

In recent years there has been considerable effort in understanding the effects of discreteness on soliton-like solutions [1], [2], [3], [4], [5], [6], [7], [8], [9], [10]. In this work we will restrict ourselves to kink solutions. Kinks connect two groundstates of a choosen system. Let us consider a nonlinear Klein-Gordon equation

$$
\frac{\partial^{2} \Phi}{\partial t^{2}}-C \frac{\partial^{2} \Phi}{\partial x^{2}}+\frac{\partial V}{\partial \Phi}=0
$$

To allow for kink solutions the potential $V(z)$ has to have at least two degenerate minima. Throughout this paper we will consider only static solutions, i.e. the field $\Phi$ will be not time-dependent. Then equation (1.1) is reduced to an ordinary differential equation

$$
-C \frac{\partial^{2} \Phi}{\partial x^{2}}+\frac{\partial V}{\partial \Phi}=0
$$

The phase space of (1.2) is two-dimensional. A kink solution corresponds to a heteroclinic orbit. This orbit connects the two hyperbolic fixed points (the groundstates) in phase space. The invariant manifolds of these fixed points overlap, according to the continuous translational symmetry of (1.1), or due to the existence of an integral of motion of (1.2).

There exist different possibilities to modify the spatial differentials in (1.2) into differences. The most common way is to represent the differences in terms of interaction forces between neighbouring particles $X_{l}$ and $X_{l-1}$ :

$$
-C\left(X_{l+1}+X_{l-1}-2 X_{l}\right)+\left.\frac{\partial V}{\partial \Phi}\right|_{\Phi=X_{l}}=0
$$

Here $X_{l}=\Phi(x=l)$, and $l$ is an integer (without loss of generality the periodicity of the discrete chain is assumed to be equal to one). Equation (1.3) is a two-dimensional symplectic map, similar to the standard map. In general the invariant manifolds of different fixed points do not overlap anymore. Instead they generally intersect in heteroclinic points at nonzero angles. The iteration of a heteroclinic point is again a heteroclinic point. One can then consider different sequences of heteroclinic points (let us call them heteroclinic orbits). All of these orbits will be exponentially attracted to the two fixed points for sufficiently large 
absolute values of $l$. Exactly two of these orbits correspond to kink solutions, and are thus related to their counterparts of the differential equation (1.2). However these two orbits have different energies (in contrast to the differential equation case). The energy difference is called Peierls-Nabarro barrier.

Let us note that there exist also choices of the difference operator such that the invariant manifolds still overlap [11]. In that nongeneric case static kink solutions exist, which can be positioned at any place on the lattice. However, the difference operators are rather unphysical, and we will not consider these nongeneric cases here.

In the limit $C \rightarrow \infty$ the two kink-type heteroclinic orbits of (1.3) approach their counterparts of (1.2). This is due to the fact, that large values of $C$ imply slow variations of these solutions as compared to the lattice spacing. Consequently it is tempting to use a perturbation approach, which links the kink solutions of (1.2) with the adjoint solutions of (1.3). In this paper we will present a first-order perturbation calculation for the heteroclinic orbits of (1.3). In section II the difference equation (1.3) is transformed into a differential equation with spatially modulated densities. This differential equation is analyzed in section III with the help of separation into slow and fast variables, such that analytical expressions for the

kink solutions of (1.3) (in first order perturbation theory) are obtained. In section IV we apply our method to two model cases and derive explicit expressions for the kink solutions. Section $\mathrm{V}$ is devoted to a discussion of the calculation of the Peierls-Nabarro energy.

\section{REFORMULATION OF THE PROBLEM}

Let us consider the following differential equation:

$$
C \Phi_{, x x}-\rho(x) V^{\prime}(\Phi)=0
$$

Here $A_{, x}$ means (partial) derivative of $A$ with respect to $x$, and $V^{\prime}$ is the derivative of the potential $V(z)$.

If we choose $\rho(x)=1$, we obtain (1.2). If we choose 


$$
\rho(x)=\sum_{l=-\infty}^{+\infty} \delta(x-l)
$$

we obtain (1.3) [12]. This is easy to see by the following reasons. First we note that $\Phi_{, x x}(l<x<(l+1))=0$ or $\Phi_{, x}(l<x<(l+1))=$ const. Thus it follows

$$
\Phi(l+1)-\Phi(l)=\Phi_{, x}(l+0.5)
$$

By integrating (2.1) from $x=l-0.5$ to $x=l+0.5$ and using (2.2) we obtain

$$
C\left(\Phi_{, x}(l+0.5)-\Phi_{, x}(l-0.5)-V^{\prime}(\Phi(l))=0\right.
$$

Combining (2.3) and (2.4) we arrive at equation (1.3), where $\Phi(l)=X_{l}$. In other words, the field $\Phi(x)$ is given by straight lines connecting its values at integer $x=l$, the field $\Phi_{, x}$ is given by a function with finite steps at integer $x=l$ and constant elsewhere, and $\Phi_{, x x}$ is a sum over delta-functions, with weights given by (2.1) using (2.2).

It is clear that one can make a continuous transition from (2.2) to (2.3) by varying $\rho(x)$ from $\rho(x)=1$ to $(2.2)$.

We rewrite (2.2) into

$$
\rho(x)=\sum_{l=-\infty}^{+\infty} \delta(x-l)=1+2 \sum_{k=1}^{\infty} \cos (2 \pi k x) .
$$

Thus we finally arrive at the following equation

$$
C \Phi_{, x x}-\left(1+2 \sum_{k=1}^{\infty} \cos (2 \pi k x)\right) V^{\prime}(\Phi)=0
$$

Note that (2.6) is still an exact reformulation of (1.3).

\section{PERTURBATION APPROACH}

Let us introduce new coordinates $x=\sqrt{C} T$ and $\Omega=2 \pi \sqrt{C}$. Then (2.6) becomes

$$
\Phi_{, T T}-\left(1+2 \sum_{k=1}^{\infty} \cos (k \Omega T)\right) V^{\prime}(\Phi)=0 .
$$


In the limit of large values of $C$ the cosine terms in (3.1) rapidly oscillate due to the increase in $\Omega$. Thus we can apply standard perturbation treatments using the separation of the field $\Phi$ into slow $\Phi^{(s)}$ and fast $\xi_{k}$ parts [13]:

$$
\Phi=\Phi^{(s)}+\sum_{k=1}^{\infty} \xi_{k}
$$

Inserting (3.2) into (3.1) and linearizing with respect to the variables $\xi_{k}$ we obtain

$$
\Phi_{, T T}^{(s)}+\sum_{k=1}^{\infty} \xi_{k, T T}=\left(1+2 \sum_{k=1}^{\infty} \cos (k \Omega T)\right)\left[V^{\prime}\left(\Phi^{(s)}\right)+V^{\prime \prime}\left(\Phi^{(s)}\right) \sum_{k=1}^{\infty} \xi_{k}\right]
$$

For the fast variables the leading order contribution yields

$$
\begin{gathered}
\xi_{k, T T}=2 \cos (k \Omega T) V^{\prime}\left(\Phi^{(s)}\right), \\
\xi_{k}=-\frac{2}{k^{2} \Omega^{2}} \cos (k \Omega T) V^{\prime}\left(\Phi^{(s)}\right) .
\end{gathered}
$$

Averaging (3.3) over the periods of oscillation of the fast variables and using (3.5) and $\sum_{k=1}^{\infty} 1 / k^{2}=\pi^{2} / 6$ it follows

$$
\begin{array}{r}
\Phi_{, T T}^{(s)}=V_{\mathrm{eff}}^{\prime}\left(\Phi^{(s)}\right) \\
V_{\mathrm{eff}}(z)=V(z)-\frac{1}{24 C}\left(V^{\prime}(z)\right)^{2}
\end{array}
$$

Note that equation (3.6) is a simple differential equation, which will be integrated for two examples in the following section.

Since we are interested in the solution of (3.1) at integer points, the arguments $k \Omega T=$ $2 \pi n$ with $n$ being an integer in (3.5). The final solution of (3.1) to first order in $1 / C$ is then given by

$$
\Phi(l)=\Phi^{(s)}(l)-\frac{1}{12 C} V^{\prime}\left(\Phi^{(s)}\right)
$$

Actually (3.8) contains also (incomplete) terms of order $1 / C^{2}$. One can expand the solution in powers of $1 / C$ and extract the first order term after solving along the given path. 


\section{TWO EXAMPLES}

\section{A. sine Gordon case}

Let us consider

$$
V(z)=1-\cos (z)
$$

The kink solution of (1.2) is given by

$$
\Phi^{(c)}(x+\alpha)=4 \arctan \left(\mathrm{e}^{x \sqrt{C}}\right)
$$

Let us consider the slow part $\Phi^{(s)}$ of the first-order perturbation. The effective potential (3.7) is given by

$$
V_{\mathrm{eff}}^{\prime}(z)=\sin (z)-\frac{1}{24 C} \sin (2 z)
$$

Consequently $\Phi^{(s)}$ is the solution of the double sine Gordon equation and can be found in [14] (note that there is an error in equation (3.7) of [14] - the sign of the power $-1 / 2$ has to be changed to $+1 / 2$ ) or can be simply calculated by integration:

$$
\begin{gathered}
\Phi^{(s)}(x+\alpha)=2 \pi-2 \arctan \left[\sqrt{1-\frac{1}{12 C}} \operatorname{cosech}\left(\sqrt{1-\frac{1}{12 C}} \frac{x}{\sqrt{C}}\right)\right], x \geq 0, \\
\Phi^{(s)}(x+\alpha)=-2 \arctan \left[\sqrt{1-\frac{1}{12 C}} \operatorname{cosech}\left(\sqrt{1-\frac{1}{12 C}} \frac{x}{\sqrt{C}}\right)\right], \quad x \leq 0 .
\end{gathered}
$$

Here $\alpha$ is an integration constant. Using equation (3.8) and expanding in $1 / C$ we finally obtain the following first-order perturbation correction for the discrete sine Gordon chain:

$$
\Phi(l)=\Phi^{c}(x+\alpha)+\frac{1}{6 C} \operatorname{sech}\left(\frac{x+\alpha}{\sqrt{C}}\right)\left[2 \tanh \left(\frac{x+\alpha}{\sqrt{C}}\right)-\frac{x+\alpha}{\sqrt{C}}\right] .
$$

Since the invariant manifolds of the two relevant fixed points of (1.3) do not overlap, but only intersect at finite angles, we have to choose the right values of $\alpha$. Clearly they are $\alpha=0$ and $\alpha=0.5$, which correspond to a kink centered on a lattice site and between two lattice sites respectively. These two possible kink solutions are known to exist for the map (1.3) [1]- 10]. 
In order to test our result we compute the exact kink solutions of (1.3) with (4.1) for different values of $C$. We use the steepest gradient method (minimization of the potential energy) and work in quadruple precision. The result will be denoted as $X_{l}$. The deviations $d_{l}$ from its adjoint solution (4.2) of (1.2) is then given by $d_{l}=X_{l}-\Phi^{c}(l)$. The perturbation approach yields $\phi(l)=\Phi(l)-\Phi^{c}(l)$ and is defined by the second term on the rhs of (4.6) . In Fig.1 we plot $d_{l}$ and $\phi_{l}$ for $C=10$ for both kink solutions ( $\alpha=0$ and $\left.\alpha=0.5\right)$. Clearly the perturbation result fits well to the exact one. In order to be more precise, we calculate the normalized squared deviation $\Delta$ of the perturbation result from the exact one:

$$
\Delta=\frac{\sum_{l=-\infty}^{+\infty}\left(d_{l}-\phi_{l}\right)^{2}}{\sum_{l=-\infty}^{+\infty} d_{l}^{2}} .
$$

Now we can evaluate $\Delta$ for different values of $C$ and see, whether it is monotonously decreasing with increasing $C$. The results for both kink solutions are shown in Fig.2. No doubt the perturbation theory gives the correct first order result.

\section{B. $\Phi^{4}$ case}

The second example is given by

$$
V(z)=\frac{1}{4}\left(z^{2}-1\right)^{2} .
$$

The kink solution of (1.2) is given by

$$
\Phi^{c}(x+\alpha)=\tanh \left(\frac{x}{\sqrt{2 C}}\right) .
$$

The effective potential (3.7) reads

$$
V_{\mathrm{eff}}(z)=\frac{1}{4}\left(z^{2}-1\right)^{2}\left[1-\frac{z^{2}}{6 C}\right] .
$$

Thus the slow part $\Phi^{(s)}$ is the solution of the $\Phi^{6}$ differential equation. It can be easily integrated using [15]:

$$
\Phi^{(s)}(x+\alpha)=\frac{\tanh \left(\sqrt{1-\frac{1}{6 C}} \frac{x}{\sqrt{2 C}}\right)}{\sqrt{1-\frac{1}{6 C} \operatorname{sech}^{2}\left(\sqrt{1-\frac{1}{6 C}} \frac{x}{\sqrt{2 C}}\right)}} .
$$


Using (3-8) we finally obtain the first order perturbation result

$$
\Phi(l)=\Phi^{(s)}(x+\alpha)-\frac{1}{12 C} \Phi^{(s)}(x+\alpha)\left(\Phi^{(s)}(x+\alpha) \Phi^{(s)}(x+\alpha)-1\right) .
$$

As in the sine Gordon case we calculate $d_{l}$ and $\phi_{l}$ and plot the results for $C=15$ in Fig.3. The normalized deviation $\Delta(C)$ is plotted in Fig.4. Clearly the perturbation theory gives the correct result.

\section{THE PEIERLS-NABARRO BARRIER PROBLEM}

Considering the success of the presented perturbation approach with respect to the kink solutions, it is tempting to use this result for calculating the Peierls-Nabarro barrier $E_{P N}$ which is given by the energy difference of the two different kink solutions. However as it was shown in [16], one has to expect that the leading order asymptotics of $E_{P N}$ contains contributions from all orders of the perturbation series for the kink solutions for large values of $C$. This is already clear by noting that the zero-order result (i.e., replacing the exact kink solution of the lattice by its counterpart of the adjoint differential equation) yields a nonzero $E_{P N}^{(0)}$. As shown in [16], these contributions are not enough to fit the exact numbers. Clearly at least the first-order perturbation result for the discrete kink has to be taken into account (yielding $E_{P N}^{(1)}$ ). But then it is clear that contributions have to expected throughout all higher orders of perturbation theory.

Let us introduce $R^{(1)}=E_{P N}^{(1)} / E_{P N}$ and $R^{(0)}=E_{P N}^{(0)} / E_{P N}$, which measure the ratio of the first order energy difference (zero order respectively) over the exact one. In Fig.5 these results are plotted for the two examples considered in the previous section. Clearly $R^{(1)}$ is much closer to unity than $R^{(0)}$, but still there exist discrepancies, which even grow with increasing $C$. This circumstance implies, that the contributions from higher orders of the presented perturbation theory in $E_{P N}$ gain more weight with increasing $C$. Consequently the first order perturbation scheme, which works as better for the kink solution as larger $C$ is, works as better for the Peierls-Nabarro energy as smaller $C$ is. So there is an intermediate 
range of values of $C$, where the first order perturbation theory can be satisfactory applied to calculate both kink shape and energy difference.

Since the calculation of the energy difference using our derived first-order perturbation results is still a matter of computing sums, it is not very practically either. Consequently the value of the presented approach is clearly in calculating analytically the first-order corrections to the kink shape (rather than the energy difference).

There exists a theoretical approach to obtain the Peierls-Nabarro energy by using properties of the splitting angle of the map (1.3) [17]. The main point is, that the asymptotic dependence of $E_{P N}$ on $C$ for large $C$ can be substituted by a form which contains higher orders. Let us briefly explain this using the sine Gordon chain as an example. The asymptotic $E_{P N}(C)$-dependence for large $C$ is given by [16]

$$
E_{P N}=Z C \mathrm{e}^{-\pi^{2} \sqrt{C}}\left(1+O\left(\frac{1}{C}\right)\right)+O\left(\mathrm{e}^{2 \pi^{2} \sqrt{C}}\right) .
$$

Here the constant $Z$ is a prefactor, and usually all perturbation approaches are aimed to fix this constant.

To give an example, let us start with the zero-order perturbation with respect to the kink shape, i.e., let us take the continuum kink solution (4.2) and calculate the energy difference $E_{P N}^{c}$ and then $Z_{c}$. Naive approaches of this particular task have in addition expanded the differences in the energy expression $\left(X_{l}-X_{l-1}\right)$ into series over differentials (see e.g. 四. Then noting that the continuum kink shape causes higher order derivatives to decay faster with increasing $C$ these higher derivatives were dropped in the mentioned naive approach. In the energy expression that means taking into account only squared second derivatives, and in the collective coordinate frame (equations of motion for the kink) it would mean taking into account only the fourth order derivative of the continuum kink shape [16]. As it was shown in [16], the dropping of higher order derivatives is wrong, since all of them contribute in leading order to $Z_{c}$. The correct result for $Z_{c}$ gives [16]

$$
Z_{c}=2^{5}\left[\frac{2}{3} \pi^{2}-1\right]+\sum_{m=2}^{\infty}(-1)^{m} \frac{2^{2 m+4} \pi^{2 m+1}}{m(2 m+1) !} \approx 237.82 \ldots .
$$


To be on the save side we plot in Fig.6 the ratio

$$
\tilde{Z}_{c}=E_{P N}^{c} /\left(C \mathrm{e}^{-\pi^{2} \sqrt{C}}\right)
$$

In the limit $1 / C \rightarrow 0 \tilde{Z}_{c}$ should approach $Z_{c}$. Clearly this is the case, demonstrating unambiguously that higher order derivatives in the continuum kink shape contribute in leading order to $Z_{c}$. To be precise the naive approach (truncating higher order derivatives) yields the result [4]

$$
64 \pi^{2} \approx 631.65 \ldots
$$

To compare with the exact result we plot $\tilde{Z}$ for the exact Peierls-Nabarro barrier in Fig.6 . Clearly the zero order perturbation fails by more than 60 percent.

Finally we plot in Fig. $6 \tilde{Z}$ for the first-order perturbation result derived in this paper. We are about 20 percent off the asymptotic behaviour. Note that both the exact and first order result for $\tilde{Z}$ are rather strongly varying with $1 / C$, so that the true asyptotic dependence (5.1) is practically never reached. Here the mentioned approach through the splitting angle [17] has clear advantages. The map (1.3) is linearized around one of its hyperbolic fixed points. The eigenvalues are given by

$$
\lambda_{ \pm}=1+\frac{1}{2 C} \pm \sqrt{\frac{1}{C}+\frac{1}{4 C^{2}}}, \quad Z_{+} Z_{-}=1 .
$$

If we replace the exponent $\left(-\pi^{2} \sqrt{C}\right)$ by $\left(-\pi^{2} / \ln \left(\lambda_{+}\right)\right)$then we can write

$$
E_{P N}=\bar{Z} C \mathrm{e}^{-\pi^{2} / \ln \left(\lambda_{+}\right)}
$$

$\bar{Z}$ is plotted in Fig.6 for the exact value of $E_{P N}$. Clearly the exact result is described by the dependence (5.5) very good even down to small values of $C$, so that: i) (5.5) is describing the true $E_{P N}(C)$ dependence much better than (5.1); ii) (5.5) is an easy to use expression; iii) the constant $Z$ can be very precisely determined by analyzing the weak dependence of $\bar{Z}$ on $1 / C$. This yields 17

$$
Z \approx 712.26 \ldots
$$


Having this number, one can determine the exact Peierls-Nabarro energy of the sine Gordon chain using (5.5) with very good precision, and down to values $C \sim 1$.

\section{CONCLUDING REMARKS}

We have derived first order corrections to the kink shape of a discrete chain. We used the methods of slow and fast variables. The resulting differential equations can be integrated explicitely, as demonstrated for two examples. Note that the presented method can be generalized to the case of anharmonic interactions as well as to time-dependent solutions. The generalization of (2.1) gives

$$
\sum_{l} \delta(x-l) \Phi_{, t t}-\left.\frac{\partial^{2} W}{\partial y^{2}}\right|_{y=\Phi, x} \Phi_{, x x}+\sum_{l} \delta(x-l) V^{\prime}(\Phi)=0 .
$$

Here $W(y)$ denotes the nearest neighbour interaction on the discrete chain $\left(y=X_{l}-X_{l-1}\right)$, which could well be anharmonic. In the examples considered above we used only harmonic interactions

$$
W(y)=\frac{1}{2} C y^{2}
$$

so that the second derivative in (6.1) simply yields $C$.

Acknowledgements

We thank S. Aubry, P. Fulde, R. S. MacKay, C. Simo, O. Usatenko and C. R. Willis for valuable discussions, and C. Simo for providing us with numerical details on the splitting angle approach. K.K. is grateful to the Max-Planck-Institut für Physik komplexer Systeme for the kind support. 


\section{REFERENCES}

[1] M. Peyrard and M. Remoissenet. Phys. Rev. B26, 2886 (1982).

[2] Y. Ishimori and T. Munakata. J. Phys. Soc. Japan 51, 3367 (1982).

[3] J. A. Combs and S. Yip. Phys. Rev. B28, 6873 (1983).

[4] V. L. Pokrovsky. J. Physique 42, 761 (1981).

[5] N. Theodorakopoulos, W. Wünderlich, and R. Klein. Solid State Communications 33, $213(1980)$.

[6] M. Remoissenet. Phys. Rev. B33, 2386 (1986).

[7] C. R. Willis, M. El-Batanouny, and P. Stancioff. Phys. Rev. B33, 1904 (1986).

[8] P. Stancioff, C. R. Willis, M. El-Batanouny, and S. Burdick. Phys. Rev. B33, 1912 (1986).

[9] C. R. Willis and R. Boesch. Phys. Rev. B41, 4570 (1990).

[10] T. Munakata. Phys. Rev. A45, 1230 (1992).

[11] J. M. Speight and R. S. Ward. Nonlinearity 7, 475 (1994).

[12] R. S. Sagdeev, D. A. Usikov, and G. M. Zaslavski. Nonlinear Physics: from the Pendulum to Turbulence and Chaos. Harwood Academic Publishers, 1988.

[13] L. D. Landau and E. M. Lifshitz. Theoretische Mechanik, Lehrbuch der Theoretischen Physik I. Akademie-Verlag Berlin, 1991.

[14] C. A. Condat, R. A. Guyer, and M. D. Miller. Phys. Rev. B 27, 474 (1983).

[15] I. S. Gradshtein and I. M. Ryzhik. Table of Integrals, Series, and Products. Academic Press (New York), 1994.

[16] S. Flach and C. R. Willis. Phys. Rev. E47, 4447 (1993). 
[17] V. F. Lazutkin, I. G. Schachmannski, and M. B. Tabanov. Physica D 40, 235 (1989). 


\section{FIGURE CAPTIONS}

Fig.1

Discrete kink shape deviations from the kink solution of the adjoint differential equation versus lattice site $l$ for the sine Gordon chain with $C=10$.

Cirlces - exact result $d_{l}$;

crosses - first order perturbation result $\phi_{l}$.

a) $\alpha=0.5$; b) $\alpha=0$.

Fig.2

The normalized deviation $\Delta$ of the first order perturbation result $\phi_{l}$ from the exact $d_{l}$ versus $C$.

Circles $-\alpha=0.5$, crosses $-\alpha=0.5$.

Fig.3

Same as in Fig.1 but for the $\Phi^{4}$ chain and $C=15$.

Fig.4

Same as in Fig.2 but for the $\Phi^{4}$ chain. 
Fig.5

The ratio $R$ of the approximated $E_{P N}$ over the exact one as a function of $C$.

Open circles - zero order result for sine Gordon chain;

filled circles - first order perturbation result for sine Gordon chain;

open squares - zero order result for $\Phi^{4}$ chain;

filled squares - first order perturbation result for $\Phi^{4}$ chain.

Lines are guides to the eye.

Fig.6

$\tilde{Z}$ versus $1 / C$ as a test for the asymptotic behaviour of $E_{P N}$ for the sine Gordon chain (see text).

Open circles - zero order result;

filled circles - first order result;

open squares - exact result;

filled squares $-\bar{Z}$ for the exact result (see text).

Solid lines are guides to the eye.

Dashed line - predicted value $Z \approx 712.26 \ldots$ for the exact result. Clearly $\bar{Z}$ is approaching

this value for $1 / C \rightarrow 0$. Also the exact result for $\tilde{Z}$ (open squares) is approaching the dashed line in the same limit.

Dotted line $-Z=64 \pi^{2} \approx 631.65 \ldots$ (see text). 
(a)

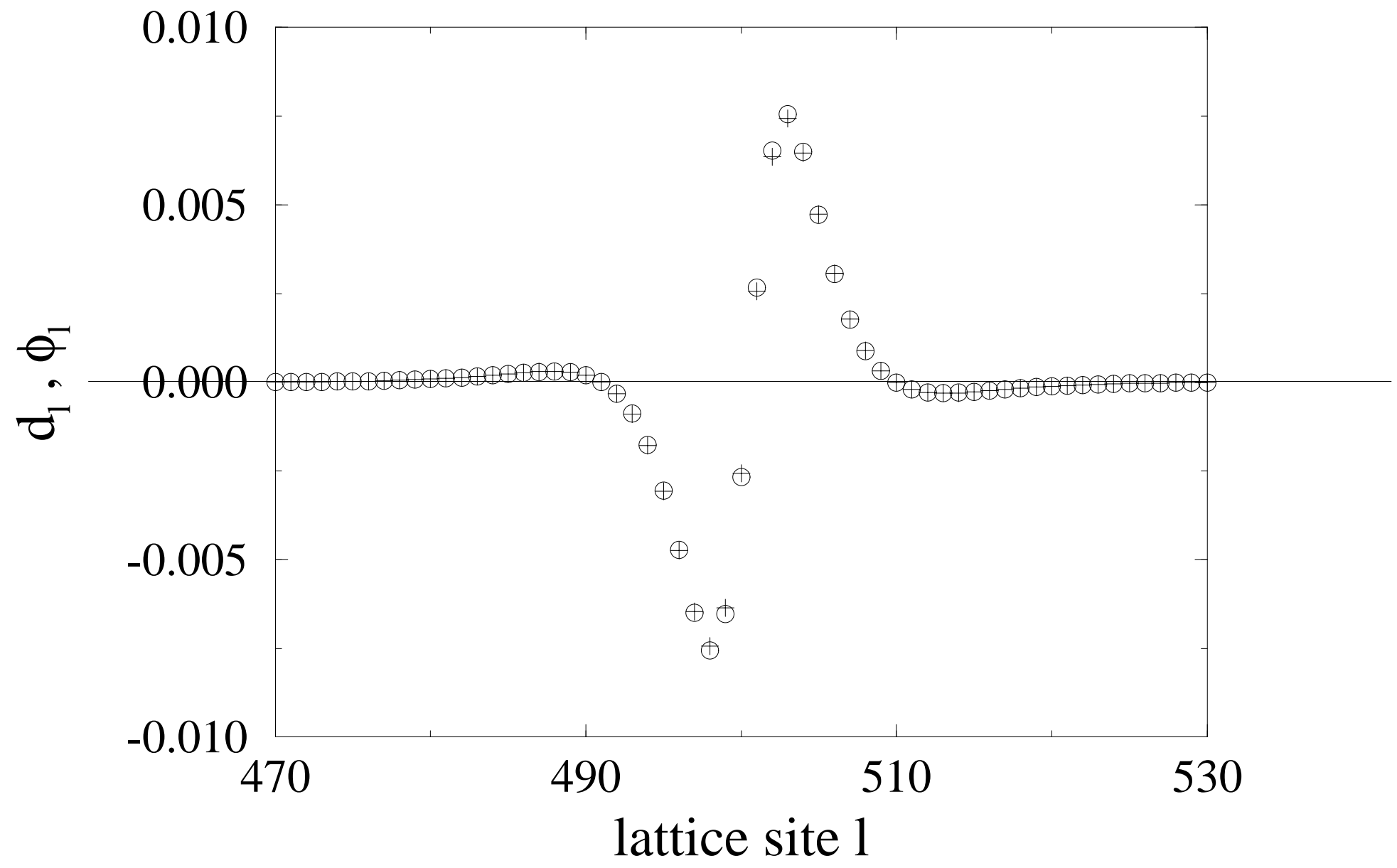

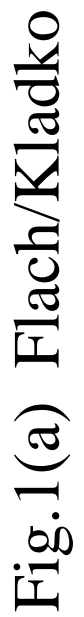




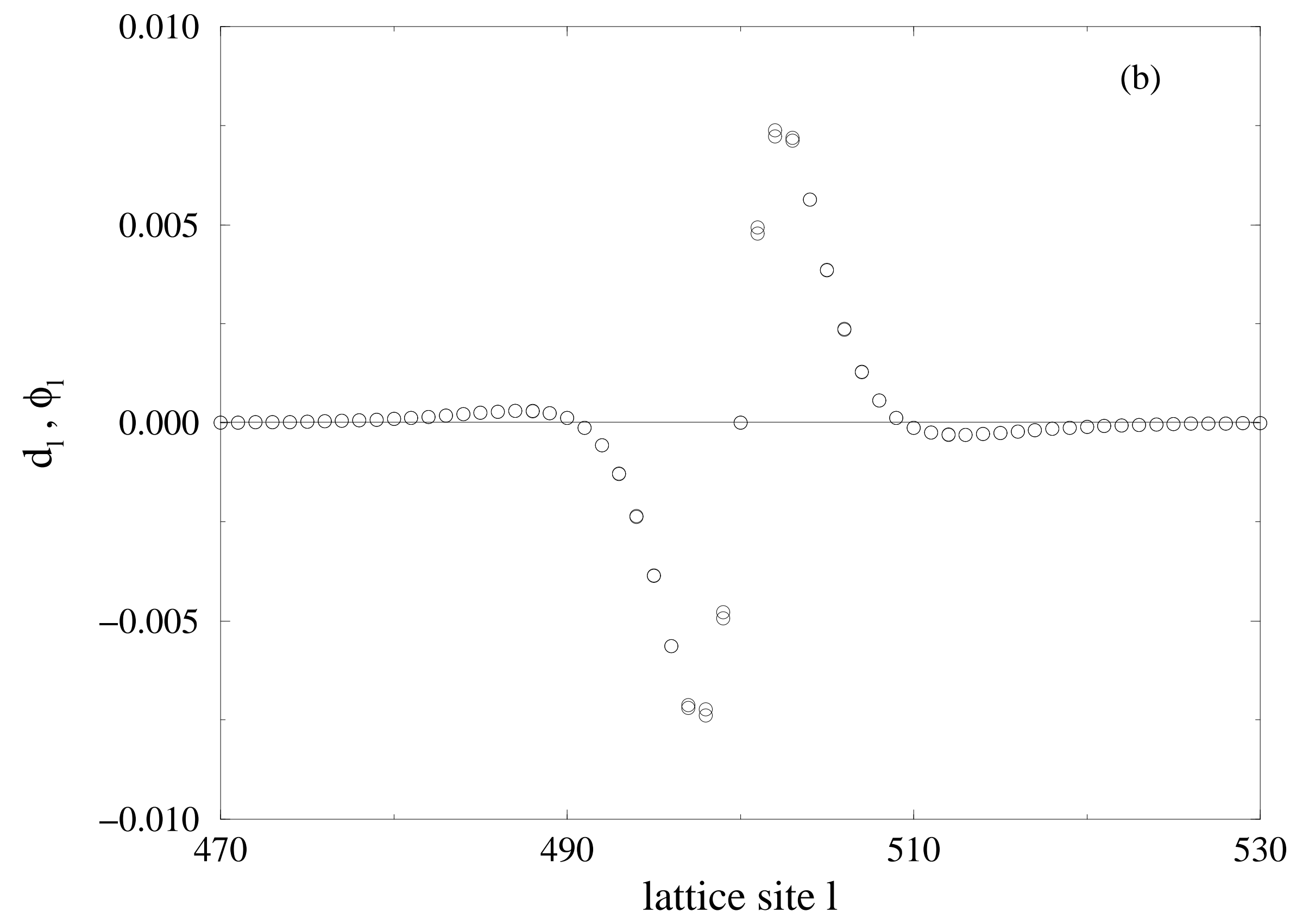

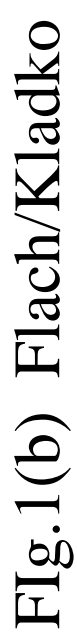




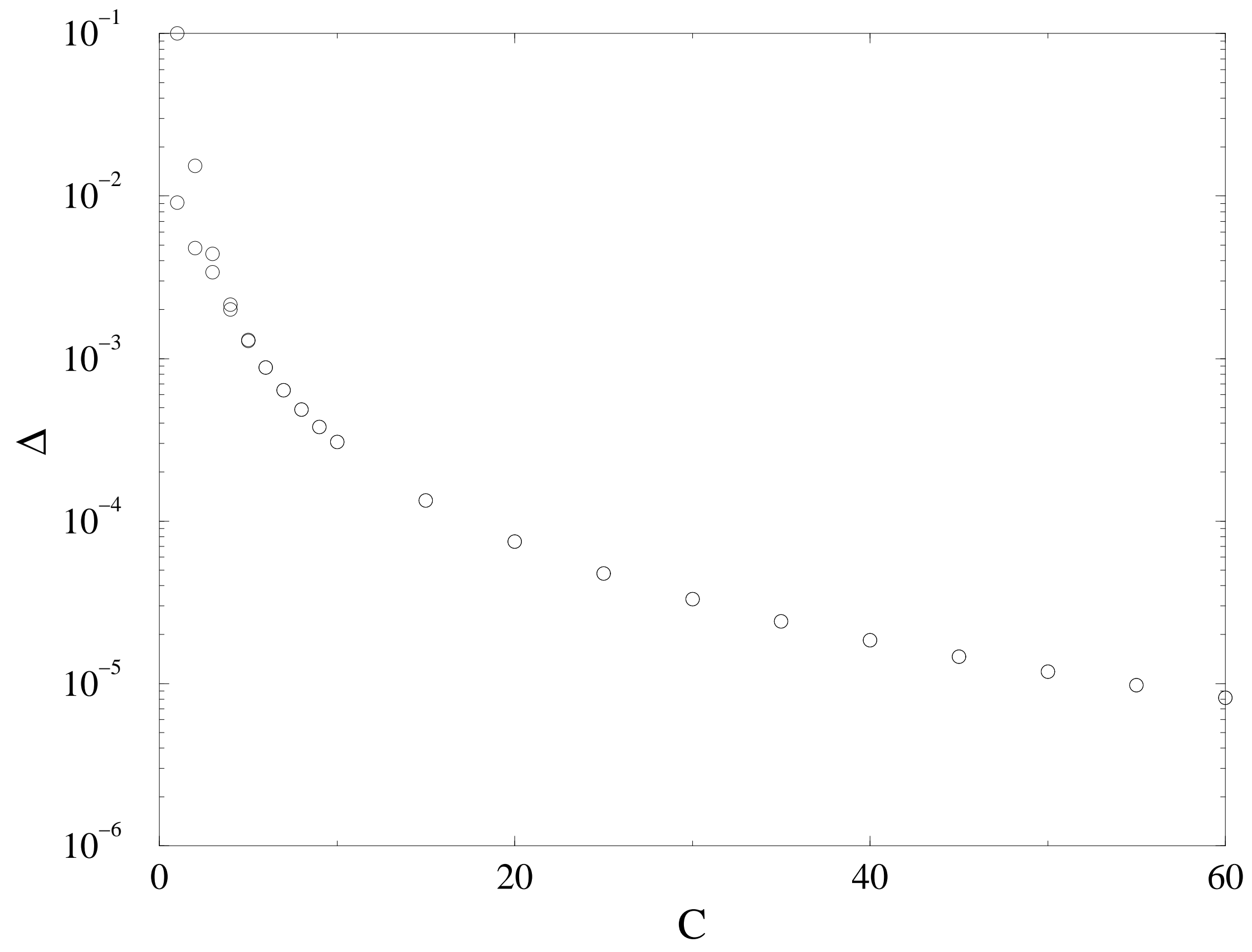

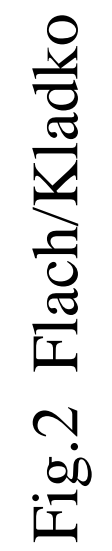




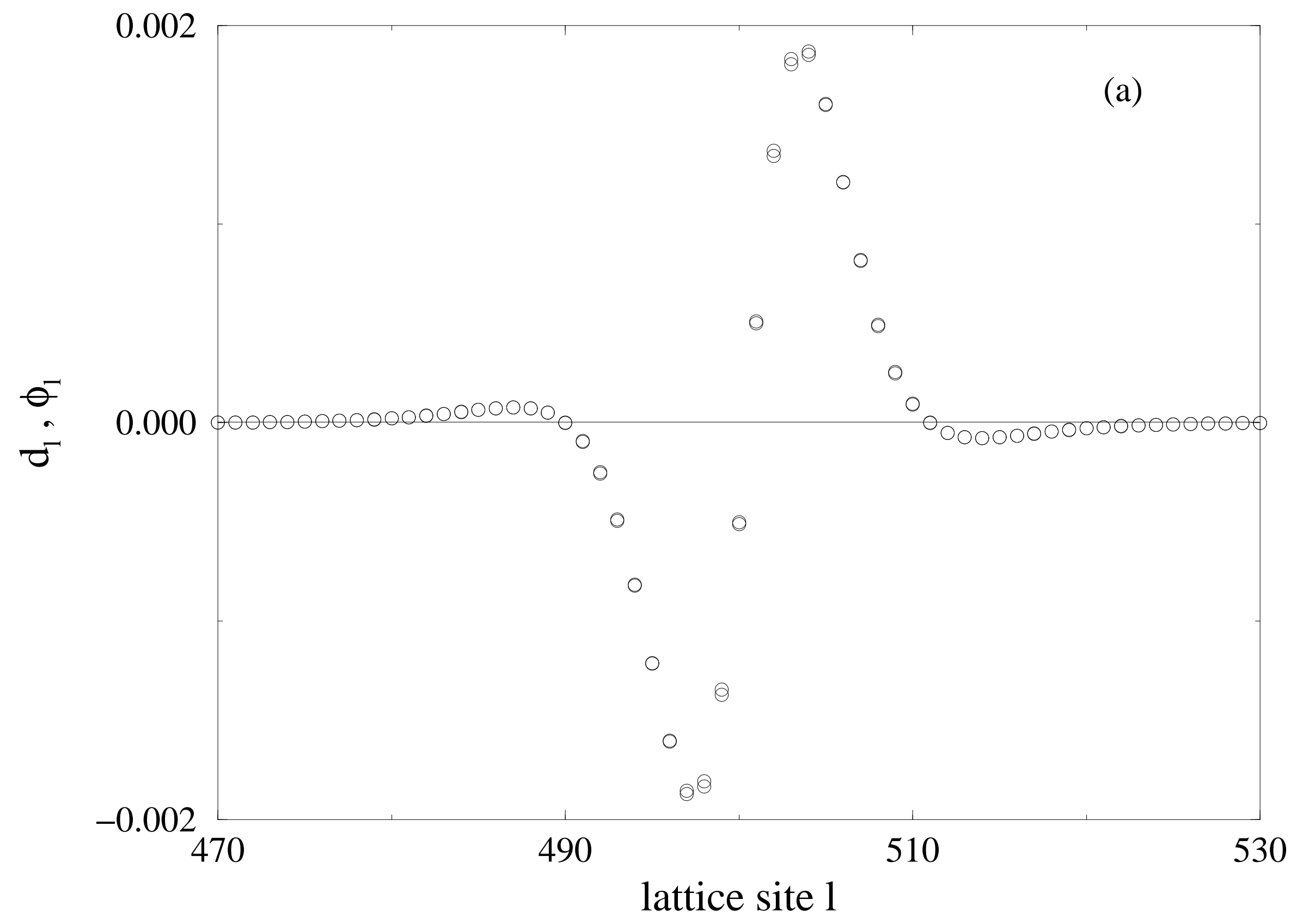

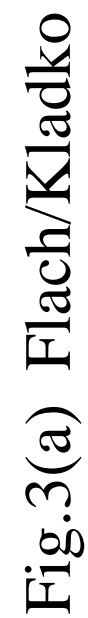




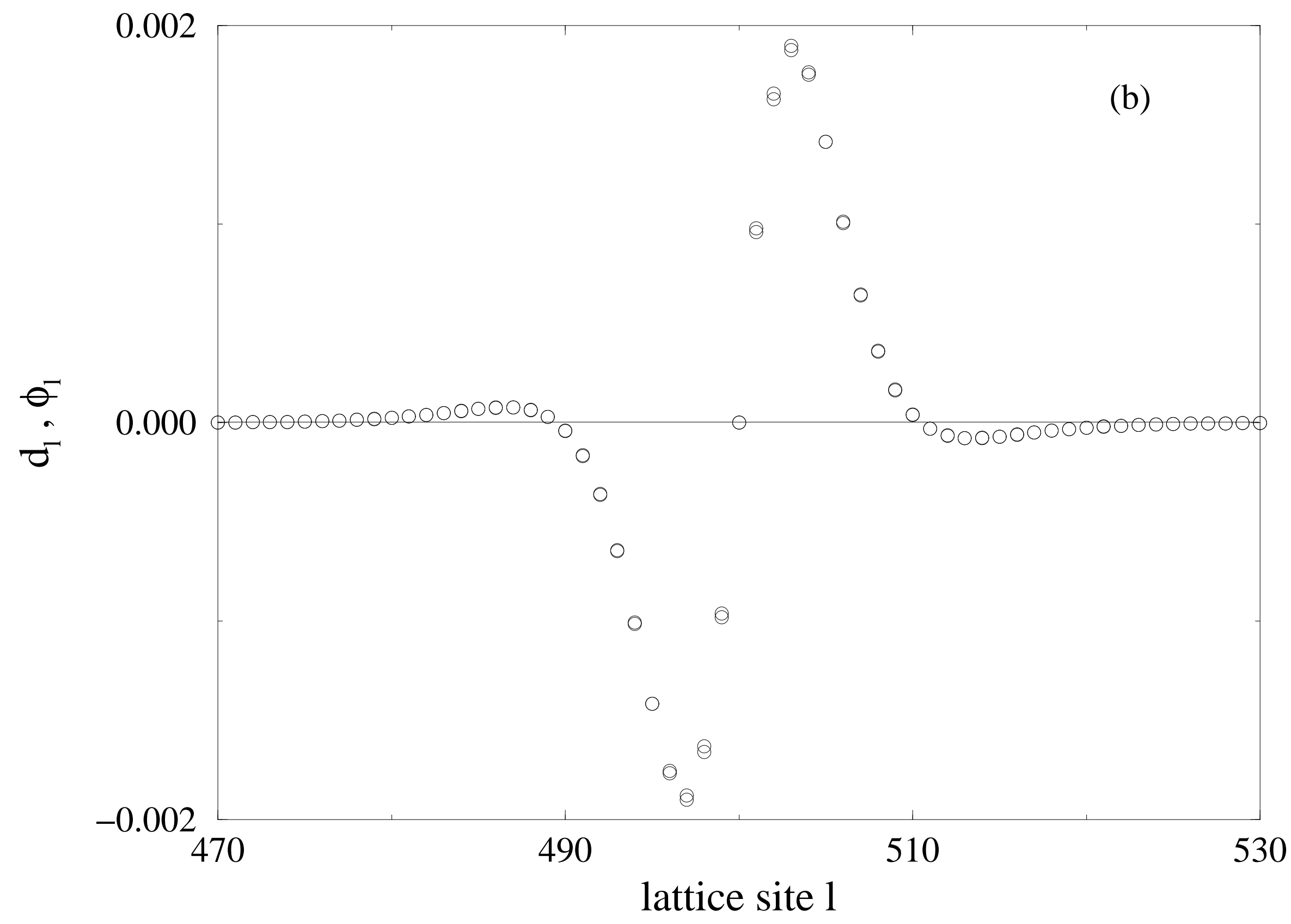

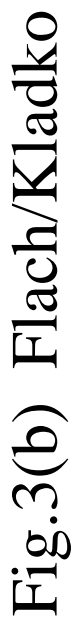




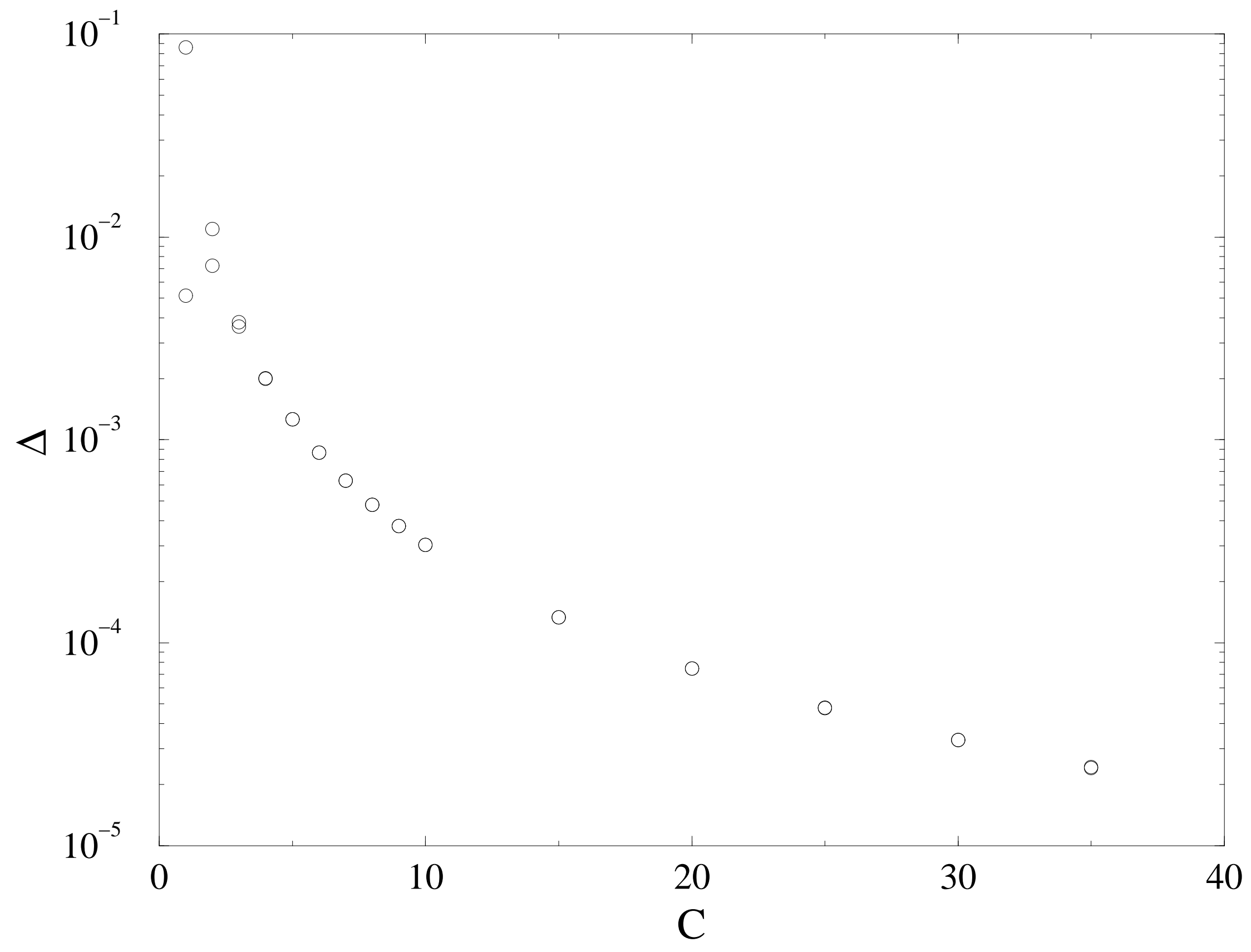

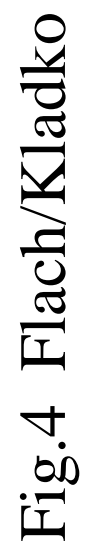

